\title{
International participation and discursive choices in the digital genre of the travel blog comment
}

\author{
Daniel Pascual ${ }^{1}$ \\ Universidad de Zaragoza \\ Departamento de Filología Inglesa y Alemana · Facultad de Filosofía y Letras · Zaragoza (Spain)
}

\section{ABSTRACT}

Current ways of informal globalized communication involve digital genres facilitating increasingly dialogical, immediate interactions. One representative example is found in blogs, where users' interaction is enabled through the genre of the comment to a previous post. In a corpus of 15 entries from 5 travel blogs, 318 comments have been analysed to study international participation in the blogosphere by different English users, native and non-native speakers. First, the quantity and length of their contributions are studied to consider the possible impact of their linguacultural background in ELF digital communication. Second, a discursive data-driven analysis of travel blog comments is provided, highlighting its most salient features, in order to better comprehend this specific digital discourse and to favour international successful participation in such a web-mediated environment.

Keywords: travel blogs; blog comment; ELF communication; digital genres; reconfigured genres

\section{RESUMEN}

Actualmente, la comunicación informal global abarca géneros digitales que promueven interacciones dialógicas e inmediatas. Ejemplo de ello son los blogs, donde la interacción de los usuarios se realiza mediante el género del comentario a un post anterior. En un corpus de 15 entradas de 5 blogs de viajes, se han analizado 318 comentarios para estudiar la participación internacional en la blogosfera de distintos usuarios de la lengua inglesa, nativos y no nativos. Se explora la cantidad y extensión de sus contribuciones para considerar el posible impacto de sus características linguaculturales en contextos de comunicación digital a través de ILF. Un análisis discursivo de los comentarios de blogs de viajes derivado de los datos enfatiza las características principales del género para comprender mejor este discurso digital y así favorecer una participación internacional efectiva en este contexto en la web.

Palabras clave: blogs de viajes; comentario del blog; comunicación a través de ILF, géneros digitales, géneros reconfigurados

\section{Introduction}

The digitalization process that the world is going through facilitates access to and availability of ubiquitous information and globalized communication among users from different sociocultural and linguistic backgrounds. This unprecedented scenario is usually enabled by the use of English as a lingua franca (ELF) (e.g. Seidlhofer 2011; Mauranen 2012), which brings together both native and non-native English speakers around discourse communities. So global, digital interactions inevitably comprise a heterogeneous group of participants, for which the English language may be their mother tongue, their L2 or even their L3. As international digital users are aware of this linguistic predominance, they attempt to spread their knowledge online through English reaching larger audiences, and giving more visibility to their ideas and texts.

Hence, boundaries between native and non-native English speakers may become blurred when aiming at global communication. International, ELF, digital interaction is increasingly gaining ground, and users with dissimilar 
backgrounds or origins simultaneously participate in communicative situations occurring online. To understand the variability and fluidity of ELF, new communicative and intercultural competences should be developed (Baker 2015), especially for those non-native English speakers. In so doing, ELF users' different communication resources and linguistic choices can be identified and studied when encountered in digital genres in this globalized, yet fuzzy, digital context.

English speakers are making use of specific digital genres to exchange their concerns, their interests and their experiences, and develop a shared sense of membership or group. They would establish themselves as a virtual discourse community: "a group of people who associate themselves over time with a computer-mediated environment, with emphasis on the social behaviour of the group" (Herring 2008: 920). When users gather online in digital genres and social media, like the blog, collaborative, bidirectional, flexible relationships may be built. This environment leans itself to participatory culture, which balances the communicative chances of both senders and recipients when it comes to being active and co-constructing the texts (Jenkins 1992; 2006). Nevertheless, participation is hardly distributed in an evenly or neutral way, since "it is constrained by market forces and hierarchies of power that interweave offline and online contexts" (Page 2012: 182). In this context, given the privileged position of English, native English speakers may be in a more advantageous position than non-native ones when it comes to participating in these international virtual discourse communities.

Therefore, it is interesting to explore this hypothesis in specific digital environments, as is the case of travel blogs, to find out whether the L1 perspective towards English predominates and how this may affect English speakers from other backgrounds. Such analyses would indicate whether there is a different nature in their participation, despite all having the potential capability of global communication. A more specific hypothesis to be tested in this study implies that non-native English speakers are more passive consumers in digital genres like the travel blog than native ones, their background affecting their individual contributions and, altogether, the level of interaction and dialogicity in the global digital discourse. In any case, analysing the digital features of specific digital genres may help international users to recognize their conventions and registers and raise awareness of the prototypical discursive practices employed in those genres. Accordingly, my research seeks to provide an answer to the following questions:

1. Are there observable differences in the quantity and length of the contributions of English international users in the genre of the travel blog comment depending on their native or non-native status towards English?

2. Which are the salient discursive and linguistic characteristics of the digital discourse employed in travel blog comments?

The remainder of this paper is divided into a theoretical framework discussing the travel blog as a reconfigured digital genre and highlighting features and affordances of the travel blog comment, a methodological section to unveil the steps taken for the linguistic analysis of travel blog comments, the presentation of findings as for English speakers participation and salient linguistic characteristics in this digital genre, and some final remarks suggesting implications of this study and future lines of research.

\section{Theoretical framework}

In this section, genre theory is introduced to better understand the nature of the travel blog by discussing its offline antecedents and its subsequent reconfiguration in the digital environment. Prominent affordances shaping the travel blog that the virtual community employs are pointed out. Then, the digital genre of the travel blog 
comment is described, as hosted in the travel blog, to understand how it is currently used online, under the belief that further research is still needed to comprehend digital users' practices when globally communicating through informal digital genres.

\subsection{The travel blog as a reconfigured digital genre}

To identify and successfully employ a genre like the travel blog, a precise communicative intent, an established audience, and an associated register or style should be recognized (Swales 2004). However, the advent of computer-mediated communication (CMC) has brought about a great deal of instability to genres, making more difficult for users to become familiar with those generic conventions. Over time, the notion of genres has moved away from regarding them as static, stable and conventional and are now conceived as much more dynamic, unstable and sophisticated (Swales 2004; Bhatia 2004). These characteristics have been further prompted by the affordances of the Internet.

Additionally, the Internet medium enables genres that are inherently interactional to promote communication or correspondence in a bidirectional way among the users employing that genre. Nevertheless, digital users' correspondents will be probably found "exclusively online, that is to say, in the guise of their 'mediatized' internet manifestations" (Mey 2018: 17). In the case of the travel blog, people meeting online share interests, wants, concerns or needs that put them together in the discourse community that typically takes advantage of a particular genre. As a digital genre, the virtual community (Herring 2008) of the blogosphere will do mostly so by using ELF, as mentioned in the introduction. They will publish their texts adhering to the generic characteristics of the travel blog, where immediacy, informality and dialogicity stand out (Pascual 2018), and they will expect them when consuming those texts. Ryshina-Pankova and Kugele (2013) claim that blogs are "combinations of interactivity and textuality, on the one hand, and of strong authorial voice and intersubjectivity, on the other hand" (185). A further key is, then, to observe and analyse the extent to which blog comments present and foster these features.

From the previous discussion, it can be inferred that the travel blog is not a simple modification of a genre to mirror itself in the Internet in the form of another genre. Instead of constituting a potential actualisation of a traditionally printed genre, like a travel article or a personal diary, it is observable that the travel blog has reconfigured, partially by taking characteristics from the mentioned printed genres. This results in acquiring new formats and portraying different communicative purposes that computer-mediated discourse analysis should explore (Herring 2013). Focusing on the conventional written-based diary, we can see the connection with the travel blog insofar as descriptive and narrative texts are intermingled in a more often than not intimate, attitudinal, but informative and general tone. Yet, although blogs are indeed interspersed with autobiographical details normally, they are meant to be read by someone else, as they are updated in an open public space that is interactive: texts can be shared, responded, flattered or criticised. This openness and loss of privacy (as the author is visible online, even if the text is anonymously published) unravels the true nature of the travel blog consisting of a communicative genre that aims to spread information -be it personal or professional, objective or subjective, structured or unplanned-and potentially exchange it.

The complex perspective that blogs offer combines the awareness of writing for a wide audience, assuming the habit of regular reflection, and inviting the audience to engage as much as they want in the communicative act through the comments, as discussed in Section 2.2. Hence, the conversational nature of the travel blog and the 
feedback provided by readers' comments broaden the dialogicity of the genre, and further indicate that the writer has the purpose of being read and answered. This leads to understand that travel blog comments show a particular structure, discourse and style dissimilar to the post they respond to. The potential functions and affordances incorporated in the reconfigured genre of the travel blog can be further analysed by exploring the digital genre of the travel blog comment within it, and its own characteristics, at the technical and the linguistic level.

\subsection{The travel blog comment}

Apart from web-dependent factors in the digital sphere such as the layout options, the prototypical length of the texts and the potential addressee, the communicative purpose can be reckoned to be the key element to identify a genre and its scope (Swales 2004; Bhatia 2004). In this regard, the travel blog, as the software genre (Lomborg 2011), can be reckoned to encompass much more nuanced generic instantiations, which should be recognised and analysed. Thus, the travel blog, whose overriding purpose is to offer and exchange knowledge, ideas and opinions among users that share interests involving the broad domain of travels and trips, permits "a wealth of functional sub-genres that can be classified according to content, directionality and style" (ibid.: 58). The coexisting genres of the travel blog post and the travel blog comment have unique communicative purposes. Whereas travel blog posts are aimed to describe to the audience trustworthy information about a place and the experiences that a person has lived there, the travel blog comment is used to show the reader's attitude towards the narrative of a post, in terms of structure, content and style. In other words, while the post is characterised by a more informational, situational and descriptive nature of a travelling experience (Pascual 2018), the comment is rather attitudinal and expressive, and presents the writers' opinions, attitudes and likes in a sort of feedback to the post. These divergences will likely lead to different discursive patterns in the two genres, and therefore unravelling particular linguistic characteristics, and their specific instantiations, in travel blog comments seem fruitful to encourage users' successful participation in a travel blog.

A generic relationship that stresses the contrast between (travel) blog posts and comments and affects the entire structure of how blogs are constructed is the genre chain, whereby some genres need to be preceded by others in a sequence to be enacted (Swales 2004). Therefore, those genres cannot be fully interpreted in isolation and, in the case of the (travel) blog, the chain entail the relationship between the post, the comment and the author's potential reply to a comment. Genres other than the post hold an unequal position and depend on the generic sequence, as authors' replies cannot be published without a previous comment, and a comment always relies on an existing post. So, the (travel) blog is "materially available to its public audience at the level of the post, where [they] may read its realized uptakes in the comments section" (Grafton 2009: 94). This relation of subordination is also reflected in the layout of the (travel) blog, as comments seem to appear embedded in the post anticipating them.

As a consequence of their communicative purposes and the genre chain, posts and comments can be assumed to be geared towards similar yet differences addressees within the virtual community: whereas bloggers' communication may not be followed in the comment section, readers publishing comments inevitably engage in a one-to-many immediate interaction, which will concern at least the blogger. These two genres and their intricacies render the level of granularity (Heyd 2016) visible within the blog. The genres hosted in it need to be observed according to their reliance and interconnection and, ultimately, the post and the comment should be comprehended as generic instances that are performed as assuming a response or being responsive (Bakthin 1986). The focus is here on the travel blog comment as a responsive genre, which will positively affect the 
dialogical nature of both blog genres. The virtual community will grow closer bonds, as comments invite them to interact, which may result in a higher degree of orality, immediacy and transparency reflected in the texts they publish and read. The working of travel blog comments fosters the audience's co-construction of the message conveyed by the digital user and, consequently, turns readers into active messengers, too (Mey 2018). This is especially interesting when happening at an international level and different speakers of English get to interact.

What is more, many of the technological features are common to the post and the comment, such as the appearance of texts in reverse chronology and layout possibilities, but others such as threaded communication are only feasible in the comments section. Web-mediated generic features are also triggered by the users' increasing needs in crafting a particular instantiation of a genre. Hence, as happens in other digital genres, affordances of the comment, and of the blog in general, "are in fact socially negotiated and therefore variable rather than fixed" (Lomborg 2011: 59). Past research in the blog has indicated that there are differences in the way common technical affordances are differently used in posts and comments. One such instance is the addition of hyperlinks, since the posts include a high amount of links to relate to other texts whereas the main point of the comment is not to offer information via hyperlinks, but to refer to other blog posts (Myers 2010). Even communicative characteristics may differ when comparing comments sections, as publication of comments in travel blogs, which is always optional, is widespread several years after the travel post publication, contrary to the tendencies regarding comments found in blogs dedicated to other topics (Goethals 2013).

So, as a situated generic instance within the travel blog, communicative practices in travel blogs should be regarded from a contextual and discursive perspective to unravel the unique and idiosyncratic characteristics of the texts. "Conversational" exchanges and social interactivity are mediated by the particular contingencies of participants, such as their sociocultural trajectories, their experiences and expectations, and the existing bonds between the communicating parties (Lomborg 2011). In this sense, how ELF communication takes place in travel blog comments is also a decisive factor in the dialogicity of the genre and how this genre responds to a previously published travel blog post. The authors' ultimate aims will be instantiated in a number of linguistic and non-linguistic features in comments that are worth analysing to better know the genre and the use of English being made.

\section{Method: corpus and procedure}

The methodological criteria followed to analyse the travel blog comment are discussed by, first, referring to the compilation of a corpus of travel blog comments and, second, explaining the procedure to analyse them in terms of users' participation and language usage.

The analysis is based on a representative corpus of 318 comments (see Table 1 below). Comments were retrieved from a corpus of 18 entries from six different travel blogs, compiled for the analysis of travel blogs (cf. Pascual 2018). The selection of travel blogs was proportionally ensured by incorporating posts published by bloggers from the three circles of English speakers: inner, outer and expanding (Bolton \& Kachru 2006), mixing users that employ English as their L1 and L2. However, one of the blogs presents no comments, which limits the number of comments sections to 15 for this study. All comments within the range of one year since the post publication were compiled. 


\begin{tabular}{lll}
\hline Circles of English & Blogs & Number of comments \\
\hline $\begin{array}{l}\text { INNER CIRCLE } \\
\text { BLOGS }\end{array}$ & Helen in Wonderlust (HW) & 46 \\
\cline { 2 - 3 } & Nomadic Matt (NM) & 78 \\
\hline $\begin{array}{l}\text { OUTER CIRCLE } \\
\text { BLOGS }\end{array}$ & The Shooting Star (SS) & 59 \\
\cline { 2 - 3 } & Zee Goes \\
\hline $\begin{array}{l}\text { EXPANDING } \\
\text { CIRCLE BLOGS }\end{array}$ & Lili's Travel Plans (LT) & 80 \\
\cline { 2 - 3 } & Girl Astray (GA) & 55 \\
\hline
\end{tabular}

Total: 318

Table 1. Information about the blogs compiled and the altogether number of comments.

To gain insights into the role users' linguacultural relationship towards English can play in a digital genre like the blog, all the comments were coded following the dichotomy native-non-native English speakers. To do so, personal information from the digital users was retrieved by tracking users' profile in the blogosphere or finding this information in the content of their comments. In cases where users' origins could not be accurately traced, comments were labelled as unidentified. Then, numbers of words per comment were counted and added to know the extension of users' contributions. Following this manual procedure, both the quantity and length of comments by native and non-native English speakers could be measured.

Next, a data-driven approach was carried out to identify salient discursive features in travel blog comments, resulting in a taxonomy of specific features -Table 2. The different levels of analysis comprise lexico-grammatical and syntactic features stemming from traditional grammars (Quirk et al. 1985; Biber et al. 1999) and metadiscourse (Hyland 2005), and a section for orthotypographic and paralinguistic characteristics, which becomes necessary when analysing digital discourse (Crystal 2006; Herring 2012; Heath 2018).

\begin{tabular}{lll}
\hline DISCURSIVE LEVELS OF & ANALYSIS AND SPECIFIC FEATURES \\
\hline Lexico-grammatical & Syntactic & Orthotypographic \\
Personal $I$ & Unfinished sentences & paralinguistic \\
Directive $Y$ ou & Question tags & Non-standard capitalization \\
Inclusive $W e$ & Copula/auxiliary deletion & Lack of capitalization \\
Attitude markers & Subject omission & Abbreviations \\
Acronyms & Non-clausal units & Onomatopoeias \\
Vague language & & Emoticons \\
Exclamations & & \\
Interjections & & \\
\end{tabular}

Table 2. Data-driven taxonomy of features in the compiled corpus of travel blog comments.

The relatively ample number of comments, and therefore speakers, may raise awareness of the nature of the travel blog comment and the type of communication within the virtual community of the blogosphere. Quantitative results were considered after manually reading the texts, in the search for possible discursive characteristics. As long as features from the taxonomy were traced once in the travel blog comments, they were counted to calculate the number of occurrences in the 318 comments of the corpus. 


\section{Results and findings}

This section presents, first, the exploration of the extent to which native and non-native English speakers may be participating in ELF communication in travel blogs. Second, quantitative results reveal the frequency and use of the features from the taxonomy to establish prototypical linguistic choices in travel blog comments. Illustrative examples from the corpus are provided to understand the saliency of these features and the potential reasons for their significance in this digital genre.

\subsection{Users' linguacultural background as a potential factor in digital communication}

Bloggers and users' background and their corresponding relationship with the English language might condition the writing of their messages and their potential publication online, thus affecting their participation. Non-native ELF speakers might, therefore, take a solely receptive role in digital dialogic genres where native English speakers are likely to read, write and interact. To observe the extent of this potential influence due to users' linguacultural origins, a closer look at the comments in the corpus was taken for each set of English speakers. Participation in this digital genre was measured in terms of contribution (number of comments published) and extension (number of words per comment) -Table 3 below.

\begin{tabular}{|c|c|c|c|c|c|c|c|}
\hline & \multirow{2}{*}{ TOTAL } & \multicolumn{2}{|c|}{ ENL speakers } & \multicolumn{2}{|c|}{ ELF speakers } & \multicolumn{2}{|c|}{$\begin{array}{l}\text { Unidentified } \\
\text { speakers }\end{array}$} \\
\hline & & $\mathbf{N}$ & $\%$ & $\mathbf{N}$ & $\%$ & $\mathbf{N}$ & $\%$ \\
\hline $\begin{array}{l}\text { Contribution: } \\
\text { Number of } \\
\text { comments }\end{array}$ & 318 & 137 & 43 & 146 & 46 & 35 & 11 \\
\hline $\begin{array}{l}\text { Extension: } \\
\text { Number of } \\
\text { words }\end{array}$ & 27706 & 10151 & 36 & 16004 & 58 & 1551 & 6 \\
\hline
\end{tabular}

Table 3. Distribution of number and percentage of comments and amount of words by English speakers in travel blog comments.

Results from the figures related to the first variable -digital users' contribution, quantifiable in the number of comments published-indicate that users' background is not necessarily determinant of their involvement in blog interaction through the publication of comments. What is more, in the corpus analysed, there is a higher number of comments authored by ELF speakers (146) than by native speakers (137). A likely explanation concerns users' anonymity allowed by computer-mediated environments when making a public contribution. It is possible not to disclose any personal information accompanying one's text thanks to the technical characteristics of the Web and the affordances of the digital genre of the blog overall. Anonymity may help speakers from different backgrounds hide their linguacultural origins and feel more comfortable when participating online in informal everyday interactions by avoiding explicit reference to their own background. Yet, such background may be revealed through inaccurate discursive anonymity: even when purposely withholding personal information, "the writing itself might reveal to a certain degree something about the message source” (Qian \& Scott 2007: 1430). This apparent blurriness in the English native-non-native distinction may actually emphasize an increase in the level of dialogicity in a digital context where all kinds of English speakers meet and interact online, as data in Table 3 display.

Results are different when looking at the second variable, extension, measuring the number of words per comment. Almost $60 \%$ of the total amount of words in the corpus correspond to comments produced by non- 
native English speakers, while native speakers roughly author more than one third of the words. Characteristics of digital genres also play a crucial role to understand the figures. The dialogicity and interactivity featuring digital genres such as the travel blog comment fosters a type of discourse which is immediate and oral. That is why messages are usually brief, precise and purposive, above all, when it comes to English native speakers' contributions. In turn, non-native speakers employ on average more words to express their messages, so apparently they need a repertoire of linguistic resources in English to develop and paraphrase their ideas in a longer stretch of discourse than native speakers tend to do.

All in all, exploring the two variables of contribution and extension of users' comments in the travel blog seems to have rejected the initial hypothesis in this paper: that non-native English speakers may take a minor role in international communication through digital genres because of their linguacultural background. On the contrary, in the corpus analysed native and non-native English speakers' contributions are rather similar. Furthermore, English non-native-authored comments are normally longer in extension than those written by English natives. This may point to a fairly equal actual presence of ELF digital users in the discussion triggered by the travel blog, regardless of their linguacultural background. This concise though illustrative study may point towards the use of ELF connecting speakers all across the globe because of their interests and concerns, in this case involving travelling and trips. The virtual community of the blogosphere seems to have raised awareness about global interactions being mediated by different kinds of English, and this is translated into a balanced participation from all kinds of English speakers in comments.

\subsection{Salient features in the genre of the travel blog comment}

To explore the nature of users' practices as regards travel blog comments, a discursive analysis of the corpus is provided to specify the salient features used by international English speakers (see Table 4 for full results). Examples are offered and implications drawn as for the type of discourse likely to be expected in this digital genre.

\begin{tabular}{lll}
\hline \multirow{2}{*}{ Specific discursive features } & \multicolumn{2}{l}{ Comments using this feature } \\
\cline { 2 - 3 } & $\mathbf{N}$ & $\mathbf{\%}$ \\
\hline Personal I & 273 & 86 \\
\hline Directive You & 234 & 74 \\
\hline Inclusive We & 86 & 27 \\
\hline Attitude markers & 305 & 96 \\
\hline Acronyms & 52 & 16 \\
\hline Vague language & 134 & 42 \\
\hline Exclamations & 214 & 67 \\
\hline Interjections & 115 & 36 \\
\hline Unfinished sentences & 87 & 27 \\
\hline Question tags & 19 & 6 \\
\hline Copula/Auxiliary deletion & 96 & 30 \\
\hline Subject omission & 114 & 36 \\
\hline Non-clausal units & 158 & 50 \\
\hline Non-standard capitalization & 71 & 22 \\
\hline Lack of capitalization & 136 & 43 \\
\hline Abbreviations & 144 & 45 \\
\hline Onomatopoeias & 67 & 21 \\
\hline Emoticons & 40 & 13 \\
\hline
\end{tabular}

Table 4. Corpus results as for the number of travel blog comments where the discursive features from the taxonomy occur and its frequency. 
The first set of features in the taxonomy involves lexical and grammatical categories authors of comments may exploit to establish interpersonal communication with the audience. Self-mentions in the form of the personal $I$, the inclusive we and the engagement marker you have been found to be common in travel blog comments for digital users to explicitly address themselves and the others when giving their opinions and following the thread of information. Not only instances of personal pronouns were counted, but also possessive adjectives and possessive, object and reflexive pronouns. Most of the comments (96\%) include individual mentions to signal authority in the text and accountability of one's beliefs. This feature is perfectly integrated in the communicative purpose of the travel blog comment, as it enables writers to distance from the audience and contribute to the discussion with their personal opinions:

(1) I am from South America but have never visited Patagonia... it is on my list. Like you I am not a lover of camping, but for views like this I would happily do camping. 2

The case of the inclusive we is also interesting, in that it may provide insights into the bonding within the virtual discourse community. At least one in four comments makes reference to a collective identity by explicitly encompassing the audience in the narration or the opinion provided. Although frequency is not particularly high, the recurrent explicit appearance of this feature evokes membership to a group in a digital asynchronous environment. In some cases either the $I$ or the we perspective is preferred throughout the whole comment (2), while in others the two self-mentions are combined to express a personal opinion about a matter affecting anyone in the audience (3). Lastly, reference to readers of one's comment by employing the directive you is also characteristic of the genre (74\%), sometimes also involving named addressees through vocatives (4). Such a feature is fundamental in travel blog comments, because "a commenter may need to pick out one of the dozens of people in a discussion for a response, or [...] to show she is responding to the original post (Myers 2010: 79). The network of connections among international users publishing comments can be further built through this mechanism, although engagement in travel blog comments from the corpus is usually geared towards the blogger authoring the post (5).

[...] It really changes us, doesn't it. And not only the kind of traveller we are but really just our whole person... It's SO rewarding! (LTNN2-8)

I find it fucking gross when travellers from relatively better developed economies collect all these experiences at the expense of others, [e]specially when we've got an international migrant crisis happening in Europe (GANN3-5)

Great post, Helen. I spent a month in Sri Lanka last year (July) and my positives are similar to yours particularly the food! [...] (HWNS1-8)

What a nice article. It's clear that you have a special fondness for San Pedro and the Atitlan area. Your photos are wonderful, not only for this story but for those that accompany all of your blogs [...] (SSNN3-9)

Attitude markers are included in almost every text analysed (96\%). They are beneficial to underline both positive and negative aspects of the sites visited and the people encountered in the usually concise descriptions and argumentations used in comments. Thus, they contribute to conveying pragmatic forces of praise or criticism

The acronyms correspond to the names of the blogs as included in Table 1 . The number that follows refers to one of the three posts compiled per blog. Last number is the number of comment in which the feature appeared. 
when sharing the travelling experience -see (6) and (7), respectively. Their instantiations are truly varied and comprise different grammatical categories such as adjectives, adverbs, and even nouns and verbs. omg Matt these pictures are amazing! (NMNS1-3)

That's too bad Greta! I can't really say I got used to it, I'll always find it disgusting, but I also think it's very eye-opening in many ways (LTNN3-2)

It is also worth commenting the case of acronyms, which, even if presenting low frequency, have two specific uses in the genre of the travel blog comment. They are employed to refer to common knowledge between the user and the audience (e.g. USA, GPS, $b \& b, Y H A, E U$ ), and to express informal or colloquial phrases that tend to be shortened (lol, OMG, ASAP, aka) or avoided in other types of discourse, like swear words ( $w t f$, af). The latter use emphasizes the potential expressive nature of the comments and is likely to have an impact on the audience, by receiving a subsequent answer (8), and so increasing the degree of dialogicity in the genre.

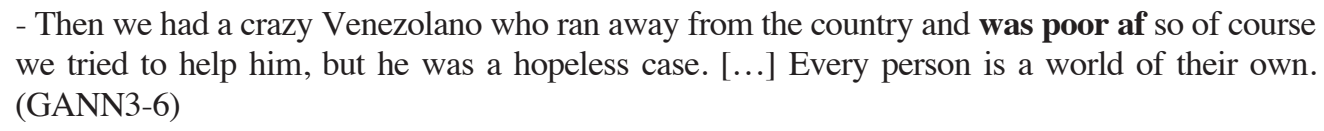
we tried to help him, but he was a hopeless case. [...] Every person is a world of their own. (GANN3-6)

- Oh my, that's just plainly rude! (GANN3-7)

Vague language is salient in comments, since almost half of them present at least one instance (42\%). Whereas bloggers might try to find precise words for their narrations, the readership may feel no constraints regarding the accuracy of their comments and the register they should stick to. As a result, in comments we can easily find vague expressions referring to a clearly defined counterpart in the post or to a collective list of items discussed by the blogger. For instance, in Nomadic Matt's post on Sydney one person publishes a comment (9) and uses the word things to allude to an ample variety of activities to do in that city, such as cultural events, coastal walks, surfing or wine tasting, as explained before by the blogger.

Oh man, I love all of these things about Sydney! (NMNS2-6)

Exclamations are also typical of the discourse of travel blog comments (67\%). Their use is intended to convey a positive effect towards the narration and its author, whether the blogger or another reader, as in Example 4, where the exclamation is initiated with an expressive acronym. What is more, exclamations also place the emphasis on striking parts within the comment, as though the writer would want the reader to pay particular attention at some point (10). Likewise, they help show the enthusiastic attitude that the virtual discourse community of travel bloggers usually adopts (11). As can be observed in this last example, exclamations are frequently accompanied by the use of interjections (36\% of comments analysed), as they many times coincide in their form and, above all, function. The supporting effect of interjections in comments is to convey a closer and more oral type of discourse than typically deployed in travel blog posts. One of the widespread uses in the corpus includes greeting the blogger at the beginning of the comment, before stating an opinion or a recommendation (12).

[...] we had the same problem in Chile, one of them followed us for miles when we were cycling! (HWNS1-1)

Oh wow, I'd love to make it to Alaska someday! (SSNN3-16) 
(12) Hey Karin, just stopped by to say thank you for the helpful article. I'm finally visiting Bratislava in a few days! Yeeey! :) (GANN1-7)

Within syntactic aspects, unfinished sentences are another characteristic featured in travel blog comments (27\%), which can be interpreted as a substitute of a hesitation or a pause in oral discourse, and not as a sort of suspensecausing sentences. They are more employed in travel blog comments than in posts, proving that comments are characterized by a greater immediacy when writing, not spending too much time on the elaboration and organization of their ideas (13). On the contrary, in posts, writers devote more time to choose the rhetorical and linguistic elements they wish to include (Pascual 2018).

Question tags present low occurrence, as they only show up in 19 comments in the corpus. They have been considered for the taxonomy of features for their rhetorical and stylistic purposes, especially when it comes to engaging the reader and seeking for certain agreement or comprehension. Subsequently, they may mean a profitable resource when writing comments, because they serve the function of calling the readership's attention (14), without actually expecting response from them. Despite a moderate presence in comments, questions tags may be fruitful for users to finish their sentences or entire messages in a straightforward way, while searching for the audience's agreement or approval when conveying their subjective opinions and the corresponding different pragmatic intents, such as praising, criticising, recommending or exaggerating.

(13) I'm afraid it's changing, too... And in some way I wish for it to change (LTNN2-3)

(14) it's just clickbait and there's ALWAYS a catch. Like "we got it for free using X million points that we happened to have for airline Y or hotel chain Z". Erhm, that's not really helpful, is it? (GANN3-26)

Subject omission (30\%) and copula and auxiliary deletion (36\%) should be regarded rather as complementary in travel blog comments, because they both alter the syntactic structure of the sentence, stressing the unorthodox register and flexible use of the language allowed and expected in electronic communication (Herring 2012). Interestingly enough, cases of subject omission, though widespread across all 15 comments sections, do not outnumber cases of copula and auxiliary deletion. This may indicate that users do not find a proper balance sometimes, and they may go from a consistent grammatical style, rather associated with travel blog posts, to completely ungrammatical rules widely accepted in some digital communicative situations, as illustrated in examples (15) and (16) below.

(15) I have no idea why I haven't been yet. Think I'll go when I have time to do a long trip! (HWNS1-6)

(16) What made you choose Georgia? (Just curious). (SSNN1-10)

Non-clausal units, that is, sentences with no verb structure whatsoever (sometimes even single words) are frequently employed in comments to greet and bid farewell to the blogger or the general audience, as well as express criticising, thanking and praising (17) in a concise but very clear way. A more special usage is to strikingly stop the line of thought expressed in the comment, somehow shocking the reader (18). Their general use in travel blog posts is dissimilar, in that they are deployed to express catchy and attractive titles and headings, close paragraphs or sections and include a caption after multimodal elements, especially pictures of the trip.

(17) This post gave me another subtle nudge back in the Cuban direction - Solid read. (LTNN2-23)

(18) I had no choice, I was in the US Army -never again (NMNS1-15) 
Concerning orthotypographic and paralinguistic features, non-standard capitalization (22\%) is an alternative way to make users' comments salient. Reasons for these characteristics to appear comprise writers' lack of revision when drafting the message (19) or the wish to emphasize a particular piece of discourse (20). In other occasions, this feature implies a pragmatic function of adding emphasis and stressing one's message to the rest of readers (21).

(19) I had to skip the desert! WHat a shame! (HWNS2-8)

(20) It's not very common here to coachsurf, at least in the South of Italy where I live (GANN3-20)

(21) Really REALLY amazing captures! I look forward to reading the next instalment and I am now subscriber ;) (LTNN1-5)

In turn, lack of capitalization at the beginning of words which require being capitalized in English seems distinctive in the genre of the travel blog comment (43\%), as perhaps when members from the virtual community are about to respond, they do not worry much about imprecision in their comments, as opposed to what happens with posts. This insufficient revision is typically encountered throughout the corpus in toponyms, demonyms and anthroponyms (e.g. sydney, portuguese, kami, respectively). Abbreviations appeared in $45 \%$ of the comments. They are also indicative of the immediate and informal discourse in travel blogs, and are regularly exploited in lexical items (22) and verbal forms which are expressed as if spoken (23). Linguistic economy and the willingness to comply with the identified discourse in travel blog comments may sometimes lead users to shorten words in particular odd ways, as in (24) which refers to Guatemala.

(22) This is such great information and great stories to go along with the info (GANN2-9)

(23) If you love Sri Lanka then you're gonna love India! (HWNS1-4)

(24) We plan on heading to Guat in the new year so Lake Atitlan goes on the agenda. (SSNN3-9)

Onomatopoeias are visible in comments $(21 \%)$, although it is one of the least exploited features within this category in the taxonomy. They entail an effective strategy to provide the discourse with a rather oral and expressive hint that entices the audience. Example (25) aims at imitating the sound a person would make when orally interacting. Additionally, onomatopoeias serve illustrative purposes, as an ornament to the information provided at a certain point of the narration or the description (26).

Lastly, emoticons are a playful discourse strategy to transmit feelings or emotions complementing the textual message. Results indicate that only $13 \%$ of the comments analysed include them, the degree of formality and closeness being key factors for their appearance. However, only one of the posts in which comments are published shows an instance of an emoticon, so this accounts for a strategic, meaningful use of the feature instead of losing its meaning and effect for being recurrently overused. This paralinguistic element enlightens the reader's reaction to the text, and reinforces the fact that the virtual community answering the blogger's post (and therefore participating in the genre chain thanks to the comments) normally does their best to show a pragmatically positive attitude. Overall, they are mostly frequent to greet and praise the author, to support users' own evaluative sentences, and to make jokes highlighting curious data. For example, a reader further admits her excitement in her comment through the emoticon in (27). 
In brief, the discourse featuring the genre of the travel blog comment is normally immediate and brief, shows oral and interactional traits to create an informal environment and admits for stylistic unorthodox and alternative ways of expression. Characteristics displaying this nature and prominently traced in the corpus include, above all, personal references $I$ and you, attitude markers, exclamations and non-clausal units. Other features should be understood as complementary in the way they may be displayed, such as subject omission and copula or auxiliary deletion, or interjections and exclamations. However, even features with lower occurrence have specific uses in the blogosphere, such as acronyms, onomatopoeias and question tags, which may be exploited by ELF speakers in particular communicative situations.

\section{Concluding remarks}

This paper has delved into the travel blog as a representative example of a non-academic prolific genre allowing frequent interaction among international English speakers because of its web features and the user's shared interests on a broad topic of popular interest such as travelling. More specifically, the analysis of the travel blog comment can allow us to draw implications regarding not only digital, global participation of different English speakers, but also its generic affordances (e.g. immediate answers to posts, inclusion of multimodal elements like pictures or videos, intentional addition of hyperlinks) and discursive characteristics (e.g. reach of wide audiences, acceptance of different registers, sense of orality when writing). These two aspects together render a certain level of interaction and rapport among bloggers and users within the virtual community, who share and produce this genre enabled by the prior publication of a post. Precisely, the predominant position of ELF, as used by English speakers of different backgrounds in the comments, may affect how communication and discourse evolve in travel blog comments.

The coexistence of English speakers from distinct linguacultural contexts has been explored. It was hypothesized that the interactional and bidirectional character of travel blogs could be exploited to a different extent by native and non-native English speakers. Results have quantitatively pointed to the fact that digital communication is not greatly affected by the users' relationship to English, as non-native English speakers' participation is not outnumbered by natives' in the corpus compiled. Actually, in the travel blog comment, non-native English speakers have been shown to comment on posts slightly more frequently and, clearly, more extensively. Nevertheless, the small size of the corpus presented, despite introducing a considerable amount of speakers, only permits to indicate that this tendency points out towards the widespread use of ELF, and needs confirmation with a bigger sample of texts to discover more representative tendencies.

Findings in this direction also reflect the complexity and unpredictability in the interaction among users participating in travel blog comments. Furthermore, the instability and dynamicity of digital genres in general, and the blog in particular, confirms the need to keep exploring other blog genres to compare the results as for users' international participation and discursive practices. This particular virtual community usage may help us comprehend, more successfully, the differences among the travel blog and other sorts of blogs.

The analysis carried out from the data-driven taxonomy has displayed that lexico-grammatical features tend to be salient in the corpus of travel blog comments (e.g. self-mentions, the engagement marker you, attitude markers, exclamations). Syntactic features are also prominent, but stand out because of the variety of linguistic choices users have at their disposal. Altering the structure of the sentence is very frequent, be it by omitting the subject, an auxiliary verb or the entire verbal phrase. At the orthotypographic level, concrete features emphasize 
the informal and sometimes careless style in digital texts. The inclusion of abbreviations and emoticons may increase the closeness and bonding among users, and resources such as non-standard capitalization reflect webmediated affordances, by which users can modify the layout of the digital text according to what they want to express. The genre of the travel blog comment has been observed to enable a high degree of informality, which is favoured in the texts by the appearance of a set of discursive characteristics (acronyms, vague language, interjections, onomatopoeias, among others). This also renders comments a more prominent position within the travel blog to ensure the dialogicity and bidirectionality of the online conversations the blogosphere are used to engaging in.

To conclude, travel blogs allow anyone with matched likes, wants and needs to meet and establish a bond inside that community. This is performed mainly through the genres of the post and the comment, which has been the object of analysis of this paper. The type of discourse employed in travel blog comments has been unravelled to understand how it is appropriately used in international scenarios, and how it can be exploited by ELF speakers, regardless of their linguacultural background. Raising further awareness of this informal digital genre derives in a conscious use of prototypical features to effectively fit users' communicative purposes and to let them engage in fruitful global interactions. This preliminary study can have implications for EFL and ESP learners, who can be better prepared to deal with a widespread and dialogical genre like the travel blog, within the context of computer-mediated communication, where English keeps being fundamental to exchange ideas in digital and international contexts.

\section{About the author}

Daniel Pascual is a PhD grantholder in the Department of English and German Studies at the University of Zaragoza. He is a member of the research group InterGEDI (Interpersonality in digital genres www.intergedi.unizar.es) financed by the Spanish Ministerio de Economía, Industria y Competitividad (FFI2017-84205-P). He is also a research fellow in the regional research group CIRES (Comunicación y Retos Sociales) funded by the Gobierno de Aragón (H16_20R). His research interests comprise computer-mediated communication, digital pragmatics, digital genre studies and discourse analysis both in academic environments, above all regarding scientific communication, as well as in non-academic and informal settings. His current doctoral project revolves specifically around the pragmatic strategies used in the academic communication of international research projects, focusing on digital media like research project websites and social media. The article presented here is part of a more holistic study around the rhetorical, intercultural and discursive exploration of blogs in informal online communication. He has published in national journals such as Journal of English Studies and international journals such as PROFILE and has presented outcomes of his research in national and international conferences.

\section{Acknowledgements}

This research was supported by the Spanish Ministerio de Economía, Industria y Competitividad (FFI201784205-P). I am also truly grateful to Dr Pilar Mur-Dueñas and Dr Rosa Lorés, members of the InterGedi 
research group, for the constructive comments and suggestions as for the structure of this paper and their support through the analysis presented here.

\section{References}

Baker, W. (2015). Culture and Identity through English as a Lingua Franca: Rethinking Concepts and Goals in Intercultural Communication. Berlin: De Gruyter Mouton.

Bakthin, M. (1986). Speech Genres and Other Late Essays. Texas: University of Texas Press.

Bhatia, V.K (2004). Worlds of Written Discourse: A Genre-Based View. London: Continuum.

Biber, D., Johansson, S., Leech, G., Conrad, S. \& Finegan, E. (1999). Longman Grammar of Spoken and Written English. Harlow: Pearson Education Limited.

Bolton, K. \& Kachru, B.B. (2006). World Englishes: Critical Concepts in Linguistics, Volume 5. London: Routledge.

Crystal, D. (2006). Language in the Internet. Cambridge: Cambridge University Presss.

Goethals, P. (2013). Travel blogs written by non-professionals: An exploratory analysis of a tourism genre. Ibérica 25, 147-170.

Grafton, K. (2009). Situating the public social actions of blog posts. In J. Giltrow \& D. Stein (Eds.) Genres in the Internet: Issues in the Theory of Genre (pp. 85-112). Philadelphia: John Benjamins.

Heath, M. (2018). Orthography in social media: Pragmatic and prosodic interpretations of caps lock. Proceedings of the Linguistic Society of American 3(55), 1-13.

Herring, S. (2008). Virtual community. In L.M. Given (Ed.), Encyclopaedia of Qualitative Research Methods (pp. 920-921). Los

Angeles: Sage.

Herring, S. (2012). Grammar and Electronic Communication. In C. Chapelle (Ed.), Encyclopedia of Applied Linguistics. Volume 6. Malden: Wiley-Blackwell.

Herring, S. (2013). Discourse in Web 2.0: Familiar, Reconfigured, and Emergent. In D. Tannen \& A.M. Tester (Eds.) Discourse 2.0: Language and New Media (pp. 1-25). Washington: Georgetown University Press. 1-25.

Heyd, T. (2016). Digital genres and process of remediation. In A. Georgakopoulou \& T. Spilioti (Eds.), The Routledge Handbook of Language and Digital Communication (pp. 87-102). London: Routledge.

Hyland, K. (2005). Metadiscourse: Exploring Interaction in Writing. London: Continuum.

Jenkins H. (1992). Textual Poachers: Television Fans and Participatory Culture. London: Routledge.

Jenkins H. (2006). Convergence Culture: Where Old and New Media Collide. New York: New York University Press.

Lomborg, S. (2011). Social media as communicative genres. MedieKultur: Journal of Media and Communication Research $51,55-71$.

Mauranen, A. (2012). Exploring ELF: Academic English Shaped by Non-native Speakers. Cambridge: Cambridge University Press.

Mey, J.L. (2018). How social is the Internet? A pragmatic view. Internet Pragmatics 1, 13-28

Myers, G. (2010). The Discourse of Blogs and Wikis. London: Continuum.

Page, R. (2015). Moving between the big and the small: Identity and interaction in digital contexts. In A. Georgakopoulou \& T. Spilioti (Eds.), The Routledge Handbook of Language and Digital Communication (pp. 403-407). London: Routledge.

Page, R. (2012). The linguistics of self-branding and micro-celebrity in Twitter: The role of hashtags. Discourse \& Communication 6 (2), 181-201.

Pascual, D. (2018). Analysing digital communication: Discursive features, rhetorical structure and the use of ELF in travel blog posts. Journal of English Studies 16, 255-279.

Qian, H. \& Scott. C.R. (2007). Anonymity and self-disclosure on weblogs. Journal of Computer-Mediated Communication 12, 1428-1451.

Quirk, R., Greenbaum, S., Leech, G. \& Svartvik, J. (1985). A Comprehensive Grammar of the English Language. London: Longman. 
Rhysina-Pankova, M. \& Kugele, J. (2013). Blog: A medium for intellectual engagement with course readings and participants. In D.

Tannen \& A.M. Trester (Eds.), Discourse 2.0: Language and New Media (pp. 183-200). Washington: Georgetown University

Press. Seidlhofer, B. (2011). Understanding English as a Lingua Franca. Oxford: Oxford University Press.

Swales, J. (2004). Research Genres: Explorations and Applications. Cambridge: Cambridge University Press. 